\title{
Can Mobility Negative Temperature Coefficient Be Reconciled with the Hopping Character of Transport in Conducting Polymers?
}

Nicolas Rolland, Juan Felipe Franco-Gonzalez and Igor Zozoulenko

The self-archived postprint version of this journal article is available at Linköping University Institutional Repository (DiVA):

http://urn.kb.se/resolve?urn=urn:nbn:se:liu:diva-164424

N.B.: When citing this work, cite the original publication.

Rolland, N., Franco-Gonzalez, J. F., Zozoulenko, I., (2019), Can Mobility Negative Temperature Coefficient Be Reconciled with the Hopping Character of Transport in Conducting Polymers?, ACS APPLIED POLYMER MATERIALS, 1(11), 2833-2839. https://doi.org/10.1021/acsapm.9boo435

Original publication available at:

https://doi.org/10.1021/acsapm.9boo435

Copyright: American Chemical Society

http://pubs.acs.org/ 


\title{
Can Mobility Negative Temperature
}

\section{Coefficient Be Reconciled with the Hopping Character of Transport in Conducting Polymers?}

\author{
Nicolas Rolland, ${ }^{\dagger, \ddagger}$ Juan Felipe Franco-Gonzalez, ${ }^{\ddagger}$ and Igor Zozoulenko*,‡ \\ †Division of Theoretical Chemistry $\&$ Biotechnology, Royal Institute of Technology (KTH), \\ SE-10691 Stockholm, Sweden \\ $\ddagger$ Laboratory of Organic Electronics, ITN, Linköping University, SE-601 74 Norrköping, \\ Sweden \\ *E-mail: igor.zozoulenko@liu.se
}

\begin{abstract}
Poly(3,4-ethylenedioxythiophene) (PEDOT) is a conducting polymer that is used in a wide range of applications such as electronics, optoelectronics and bioelectronics, where the fundamental understanding of the charge transport, and in particular of the electrical conductivity $\sigma$, is a prerequisite to develop new high performance devices. There are many reports in the literature where the conductivity of archetypical conducting polymer PEDOT doped with Tosylate (PEDOT:TOS) exhibits a negative temperature coefficient, $\frac{d \sigma}{d T}<0$, which is strikingly different from the activated-type behavior with $\frac{d \sigma}{d T}>0$ commonly observed in most conducting polymers. This unusual temperature dependence was attributed to the transition from the photon-assisted hopping to the metallic behavior, which is however difficult to rationalize taking into ac-
\end{abstract}


count that this transition occurs at high temperatures. In order to understand the origin of this unusual behavior, a multi-scale mobility calculations in PEDOT:TOS for the model of hopping transport were performed, where changes in the morphology and the density of states (DOS) with the temperature were explicitly taken into account. The morphology was calculated using the Molecular Dynamics simulations, and the hopping rates between the chains were calculated quantum-mechanically following the Miller-Abrahams formalism. Our results reproduce the observed negative temperature coefficient, where however the percolation analysis shows that this behavior mainly arises because of the changes in morphology upon heating when the system becomes less ordered. This results in a less efficient $\pi-\pi$ stacking and hence lower mobility in the system. We therefore conclude that experimentally observed negative mobility temperature coefficient in conducting polymers at high temperatures is consistent with the hopping transport, and does not necessarily reflect the transition to a metallic band-like transport. Based on our multi-scale modeling we introduce a simple Gaussian Disorder Model for the efficient mobility calculations, where the DOS broadening is a function of the temperature, and where the transfer integrals distribution is a bimodal distribution evolving with temperature.

\section{Keywords}

PEDOT, Electrical mobility, Negative temperature coefficient, Hopping transport, Band transport, Multiscale calculation

\section{Introduction}

Understanding and modelling charge transport in conducting polymers is the key for a fast and systematic design of new high performance polymeric materials. ${ }^{1}$ In particular, Poly(3,4-ethylenedioxythiophene) (PEDOT) has been the subject of a very large number of publications due to its remarkably good properties, ${ }^{2}$ with, among others, a relatively high 
conductivity and Seebeck coefficient that makes it an ideal material for a wide range of applications. With the urgent need for clean energy, there has been a great focus on devices capable of convert wasted heat directly into electricity. Here, PEDOT doped with Tosylate (PEDOT:TOS) has been recently considered as a promising candidate for electrode material in thermogalvanic cells ${ }^{3}$ or as a thermoelectric material ${ }^{4}$, and thus control over the electrical and thermoelectric properties of this material is crucial. The conductivity $\sigma(T)$ in pristine PEDOT films as well as in most other conducting polymers usually shows an activated-type temperature dependence associated with the phonon-assisted hopping transport, $\sigma(T)=\sigma_{0} \exp \left[-\left(W / k_{B} T\right)^{\gamma}\right]$, where $\sigma_{0}$ is a pre-factor, $W$ is an activation energy, and $\gamma$ is a power that depends on the transport dimensionality or interpretation of hopping. ${ }^{5-10}$ This behavior leads to the positive temperature coefficient (TC) $\frac{d \sigma}{d T}>0$, where the conductivity (or mobility) increases with temperature. Pretreatment and/or post-treatment with various organic solvents, salts, and acids that are used in order to increase $\sigma$ (referred as secondary doping process ${ }^{11}$ ) can drastically affect the $\sigma$ temperature dependence. Kim et $a l .{ }^{8}$ demonstrated a 500-fold conductivity enhancement in PEDOT doped with poly(styrenesulfonate) (PSS) by adding a polar solvent and a subsequent solvent bath treatment. They claimed that the polar solvent increased the interchain packing and reduced the disorder, inducing an insulator-to-metal transition (IMT) as suggested by the positive slope encountered in the reduced activation energy plot $W=d \log (\sigma) / d T$ versus $T$ ( $W$-plot). ${ }^{12}$ In this case, the transport model had to be changed from the Variable-Range-Hopping to the FluctuationInduced Tunnelling model ${ }^{13}$ to fit the $\sigma(T)$ curves, where $\sigma$ extrapolates to a finite value at $0 \mathrm{~K}$, like a metal, but still with a positive TC. Farka et al. ${ }^{14}$ applied high pressures to PEDOT films doped with sulphate to induce denser chain packing and also observed an apparent MIT in the $W$-plot and a finite $\sigma$ at 0K. In addition, they observed a negative TC at low temperatures, below 10K. A similar behavior was also reported by Massonnet et al. ${ }^{15}$ for PEDOT doped with trifluoromethanesulfonate (OTf) with additionnal sulfuric acid treatment. Several other studies also reported a negative TC at high temperature, above 
300K, for various PEDOT based materials. ${ }^{16-19}$ By a dopant engineering approach using OTf as the oxidant, N-methyl pyrrolidone as polymerization rate controller and sulfuric acid as dopant, Gueye et al. ${ }^{20}$ were able to manufacture highly conducting PEDOT films (5400 $\mathrm{Scm}^{-1}$ ) where both low and high temperatures negative TC were observed . In this case, an heterogeneous model consisting of metallic islands, the polymer crystallites, dispersed into a disordered matrix where MIT can occur, successfully fitted the $\sigma(T)$ curves. The low temperature negative TC is attributed to the conductivity being dominated by that of a disordered metal (matrix in the metal side of the MIT), while quasi-1D metallic conduction in the crystallites is supposed to dominate conductivity in the range of the high temperature negative TC. ${ }^{21}$ The negative TC over the entire temperature range of observation has been reported for well-ordered PEDOT films doped with Tosylate (TOS). ${ }^{22}$

All the aforementioned studies have in common that they attribute a change in the $\sigma$ temperature dependence to a change in the transport mechanism, assuming that the hopping transport (with positive TC) changes into metallic band-like transport (with negative TC). This assumption is reasonable for the case when the negative TC is observed at very low temperatures followed by the positive TC at elevated temperatures ${ }^{14,15,20,23}$, which is interpreted as a transition from the metallic to the hopping transport as temperature is increased. However, the transition from the hopping to the metallic behavior as temperature is increased is difficult to rationalize. Indeed, in the metallic regime at elevated temperatures the scattering is caused by the phonon scattering. As a number of phonons is proportional to the temperature $\mathrm{T}^{24}$ the metallic conductivity decreases with decrease of $\mathrm{T}$. At the same time, the hopping conductivity (which is assisted by phonons) is instead enhanced. In addition, there is one more important factor that is often overlooked. This is the phase coherence length $l_{\phi}$, which should be large enough (at least comparable to a size of polymer crystallites) in order to sustain the metallic behaviour. Note that $l_{\phi}$, decreases with temperature due to inelastic phonon scattering. The phase coherence length was the focus of numerous studies for metals and semiconductor heterostructures ${ }^{25}$. For example, in GaAs heterostructures, 
which are characterized by perfect crystallite order and mobilities exceeding those of PEDOT by $4-5$ orders of magnitude, $l_{\phi}$ is of the order of $1000-100 \mathrm{~nm}$. The reports on $l_{\phi}$ in conjugated polymers are scarce. Recently, Kang et al. reported $l_{\phi}$ decreasing from $5 \mathrm{~nm}$ to $2 \mathrm{~nm}$ in the temperature range $10 \mathrm{~K}-120 \mathrm{~K}$ in a highly ordered thiophene-based conjugated polymer ${ }^{26}$. This value of $l_{\phi}$ is sufficiently large to sustain the metallic behaviour in crystallites consisting of several chains. At the same time, for the case of much more disordered polymer PEDOT:PSS, the authors of Ref. 26 were not been able to extract of $l_{\phi}$, which was taken as a signature of the wave function localization within a single polymeric chain, and therefore predominantly hopping transport regime. Also, in the past, a negative TC observed at high temperatures were systematically attributed to the existence of metallic islands in the material, a model that was built more than 20 years ago based on morphologies of long polymer chains (hundreds of monomers) forming very large fibrillar crystallites where the chains can fold back in the same crystallite or bridge several crystallites; ${ }^{27}$ it is however unclear how this model can be applied to PEDOT consisting of short chains ( $<20$ monomers). ${ }^{28,29}$

It is generally recognized that the morphology of PEDOT plays a decisive role in the determination of its transport properties. ${ }^{2,30}$ At the same time, there have been no attempts in the past to relate the change of the temperature dependence of the conductivity to the change in the morphology, as opposed to the change in character of the transport. On the other hand, it is now possible to probe the relationship between morphological structure and carrier mobility with multi-scale calculations of conducting polymers and related systems, ${ }^{30-37}$ where the material morphology is obtained with Molecular Dynamics (MD) simulations corresponding to a given set of conditions, e.g. temperature, dopant concentration, presence of additives. In this letter, we perform such multi-scale calculations of the charge mobility as a function of temperature in a PEDOT:TOS film, where the changes of the morphology and the density of states as the temperature increases are explicitly accounted for. MD simulations of the material where the temperature is progressively changed are performed, then Quantum Mechanical (QM) calculations of the transfer integrals (TI) and localized states 
energies are performed for several snapshots of the morphology obtained from the MD simulations, and finally the carrier mobility is calculated using the same snapshots. Within the present multi-scale model, we are thus able to investigate the temperature dependence of the mobility by taking into account temperature induced morphological changes that are totally eluded in standard hopping models such as the VRH model. We demonstrate that when these morphological changes are accounted for, the hopping mechanism can result in the negative temperature dependence of the mobility. Based on our multi-scale calculations, we propose an extension of the so-called Gaussian Disorder Model (GDM) that provides an efficient way for the mobility calculations in the conducting polymers.

\section{Method}

The bulk morphology of the system was generated by atomistic Molecular Dynamics (MD) using similar parameters as in our previous works addressing the morphology of PEDOT:TOS. ${ }^{30,36,38}$ It should be stressed that the utilized approach is proven to be in the position to accurately describe and predict the polymer morphology and achieve a quantitative agreement with experiment. This includes, for example, the X-ray diffraction pattern reproducing the degree of crystallinity $\left(\pi-\pi\right.$ stacking and number of chains in crystallites for PEDOT ${ }^{38,39}$, and related polymers ${ }^{40}$; formation of a granular structure in PEDOT:PSS ${ }^{39,41}$; formation of lamellar structure and preferential edge-on orientation on the substrate ${ }^{36}$; mobility dependence on the chain length ${ }^{30}$; change in the morphology at high oxidation levels ${ }^{19}$. We simulated the evolution of a mixture of PEDOT chains and TOS ions during a progressive cooling of the material (more details can be found in the SI). Four different realizations of the initial simulation box (i.e. random positions of the molecules in the box) were produced to reduce statistical fluctuations, and for each of them we analyze the morphology on several frames corresponding to equilibrated systems at temperature ranging from $800 \mathrm{~K}$ to $100 \mathrm{~K}$ with a step of 50K (representative morphologies during the cooling are shown in Figure S1 of 

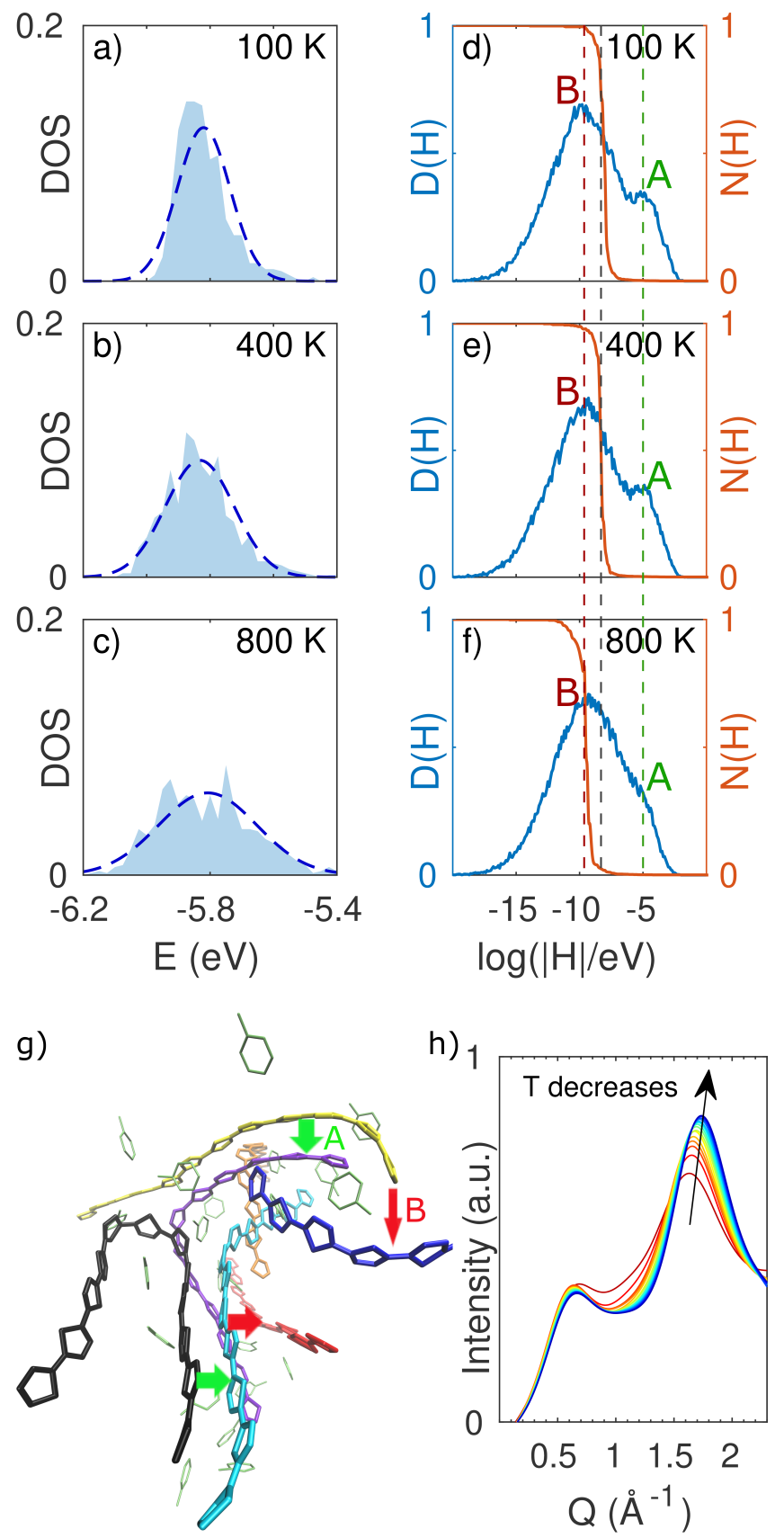

Figure 1: DOS calculated using HOMO energies distribution at (a) $\mathrm{T}=100 \mathrm{~K}$, (b) 400K, and (c) $800 \mathrm{~K}$ (shaded areas) and the Gaussian fits (dotted lines). (d),(e),(f) Transfer integral distribution $D(H)$ (blue lines) and percolation curves $N(H)$ (orange curves) at $(\mathrm{d}) \mathrm{T}=100 \mathrm{~K}$, (e) $400 \mathrm{~K}$, and (f) $800 \mathrm{~K}$. Peaks A and B in (d)-(f) corresponds to the hopping in misaligned and $\pi-\pi$ stacked configurations as illustrated in $(\mathrm{g})$. The vertical dotted lines are guides for the eyes. (g) Representative part of the PEDOT:TOS morphology at 100K. Only PEDOT backbones and TOS rings (green) are shown for clarity. A and B respectively highlight $\pi-\pi$ stacking and misaligned configurations. (h) Calculated XRD diffraction patterns as a function of temperature, from $800 \mathrm{~K}$ (red curve) to $100 \mathrm{~K}$ (blue curve.) 
the SI). Carrier mobility was subsequently calculated for the various temperatures following the model developed in our previous works. ${ }^{30,36}$ (Note that the thermal stability of PEDOT thin films is lower than $800 \mathrm{~K}$. In our calculations we use this wide temperature interval to be able to clearly trace the temperature dependence of the mobility). In this model, a network of transport units that can each accommodate one charge carrier is built based on the PEDOT chain positions obtained from MD. These transport units represent localized states in the material, and their energies are randomly drawn from a Gaussian DOS with the broadening $\Sigma$. The transfer integrals between transport units are then calculated by QM calculation using the morphology of PEDOT chains as obtained from the MD simulations (see SI and Ref. 30 for details). Eventually, the hopping rates between two transport units are calculated following the Miller-Abrahams formalism: ${ }^{42}$

$$
\omega_{i j}=\omega_{0} \times\left|H_{i j}\right|^{2} \times \exp \left(-\frac{E_{j}-E_{i}+\left|E_{j}-E_{i}\right|-2 e \vec{F} \cdot \overrightarrow{r_{i j}}}{2 k_{B} T}\right),
$$

where $\omega_{0}$ is a pre-factor, $H_{i j}$ is the transfer integrals between molecular orbitals representing

the localized states $i$ and $j, E_{i}$ and $E_{j}$ are the states energies, $\vec{F}$ is the applied electric field and $\overrightarrow{r_{i j}}$ is the vector between sites $i$ and $j$. The carrier mobility is finally obtained by solving the Master Equation for transport in the mean field fashion as introduced by Yu et al. ${ }^{43} \mathrm{In}$ this letter, we also investigated how the Seebeck coefficient evolves with temperature. When the Master Equation for transport is solved, it is obtained by $S(T)=\left(E_{F}-E_{\text {Trans }}\right) /(|e| T)$ where $E_{F}$ is the Fermi level, $E_{\text {Trans }}$ is the transport energy, $e$ is the electron charge and $T$ is the temperature. ${ }^{44}$ More details on how we calculate $E_{F}$ and $E_{\text {Trans }}$ can be found in the SI.

\section{Results and discussion}

To provide further insight on how the different transport parameters, namely the DOS broadening $\Sigma$ and transfer integral distribution, impact the mobility temperature dependence, we calculate the mobility for four different versions of the model. We first calculated the mo- 
bility based on the MD morphology at the fixed temperature $T=300 K$, with the fixed DOS broadening $\Sigma_{300}=\Sigma(T=300 K)$, where the temperature dependence of the mobility is only due to the temperature dependence of the Miller-Abrahams hopping rates (Equation 1 ); hereafter we refer to this version as the $H_{300} \Sigma_{300}$ model. The broadening $\Sigma$ is actually really cumbersome to examine in such a complicated system. ${ }^{45,46}$ In principle, it originates both from the PEDOT chain conformations and the counterions distribution within the bulk, that intuitively should be both sensitive to the temperature. It is generally accepted that the DOS can be approximated by a Gaussian distribution with typical standard deviation $0.1 \mathrm{eV} \cdot{ }^{30,44}$ Therefore, we set $\Sigma_{300}=0.1 \mathrm{eV}$. In a second version, $H_{300} \Sigma_{T}$, we still calculate the mobility based on the MD morphology at the fixed temperature $T=300 \mathrm{~K}$, but let $\Sigma$ evolve with temperature. Again, we have not direct way of calculating this evolution. Therefore, we approximate the derivative $d \Sigma / d T$ by the quantity $d \Sigma_{H O M O} / d T$, where $\Sigma_{H O M O}$ is the broadening of the distribution of the HOMO level energies in the system (as obtained from the QM calculations on single neutral PEDOT chains obtained via MD). Figure 1(a)-(c) shows these distributions together with a Gaussian fit at three different temperatures. There is a clear broadening of the distribution due to the thermal agitation of the chains, and analysis of the broadening shows a linear evolution of $\Sigma_{H O M O}$ with temperature, with $d \Sigma_{\text {HOMO }} / d T=8.88 \times 10^{-5} \mathrm{eV} / \mathrm{K}$ (Figure S2 of the SI). Therefore, we set $\Sigma(T)=\Sigma_{300}\left(8.88 \times 10^{-4} T+0.73\right)$. In a third version $H_{T} \Sigma_{300}$, we turn off the evolution of $\Sigma$ with temperature, but we account for the change of the morphology with temperature in the calculations of the transfer integrals $H$. And finally, the fourth version $H_{T} \Sigma_{T}$ takes into account both the temperature evolution of the DOS and the transfer integral distributions.

Impact of $\Sigma$ and $H$ on the temperature dependence of the mobility is reported in Figure 2 for four different versions of the model of the mobility calculation. Not surprisingly, the $H_{300} \Sigma_{300}$ model (Figure 2a) exhibits a thermally activated conductivity typical of hopping transport, as highlighted by the plot in logarithmic scale in the inset. The $H_{300} \Sigma_{T}$ model (Figure 2b) shows an activated behavior followed by a slightly negative TC at high temper- 

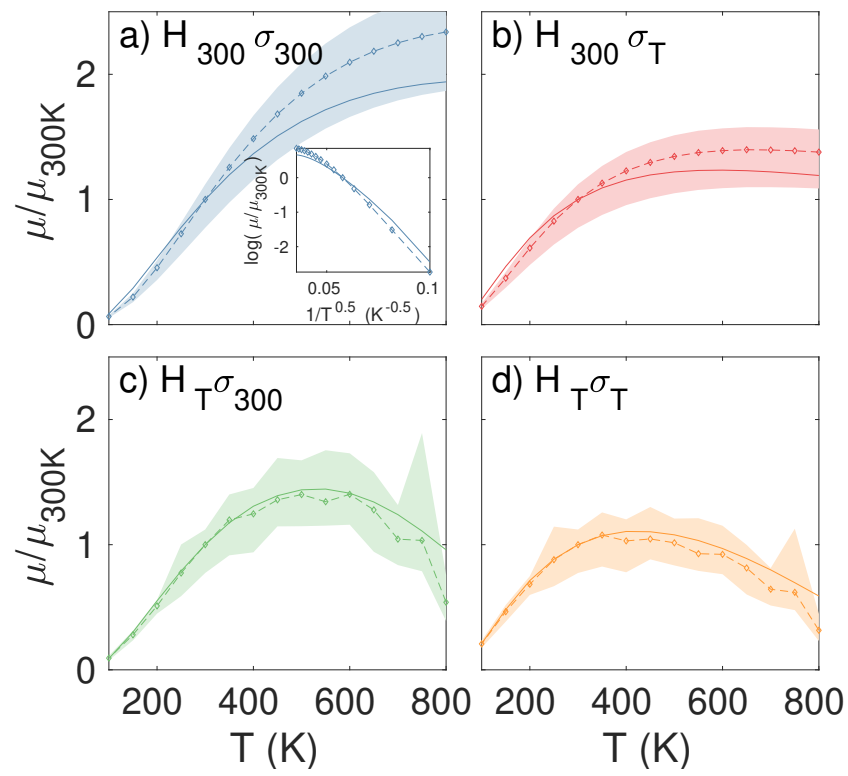

Figure 2: Mobility as a function of temperature for four different flavors of the model, (a) $H_{300} \Sigma_{300}$, (b) $H_{300} \Sigma_{T}$, (c) $H_{T} \Sigma_{300}$, (d) $H_{T} \Sigma_{T}$ (symbols and dotted lines show average values over $4 \mathrm{MD}$ snapshots for the 4 different morphology realizations, shaded areas show extremum values) and for the generalized GDM (solid lines).

atures. It is the result of the competition between increase of mobility due to temperature activated hopping and concomitant decrease of mobility due to increased disorder $(\Sigma)$ with temperature. Understanding the impact of $H(T)$ on mobility as found in the $H_{T} \Sigma_{300}$ model (Figure 2c) necessitates to investigate the evolution of the TI distributions with temperature. This is shown in Figure $1(\mathrm{~d})$-(f). At high temperature $T=800 K$, the distribution essentially consists of one peak corresponding to moderate TI values, around $\log (|H| / \mathrm{eV} \simeq-10)$. When the temperature is decreased down to $T=100 K$, there is a progressive apparition of a second peak at high TI values, around $\log (|H| / \mathrm{eV}) \simeq-5$. These peaks in the TI were attributed to hopping between misaligned chains (peak B) and hopping between chains in the $\pi-\pi$ stacking configuration (peak A) ${ }^{30}$ (hopping B and A as illustrated in Figure 1g.) This is reflected in the calculated X-ray diffraction (XRD) patterns during the cooling, see Figure 1 h. The XRD patterns exhibit a pronounced peak at $Q \simeq 1.8 \AA^{-1}$ corresponding to the $\pi-\pi$ stacking, ${ }^{30,38}$ which becomes higher and narrower during the cooling. According to the Scherrer equation, the mean size of the crystallites is inversely proportional to is the 
full width at the half-maximum of the peak ${ }^{38}$. Hence, the crystallites become larger upon cooling This behavior is consistent with the apparition of the $\pi-\pi$ stacking peak in the TI distribution. We attribute these morphological changes to a local reorganization of the chains during the cooling: overall, the global morphology is unchanged with temperature, but locally chains that are close to each other can reorganize to form well-defined $\pi-\pi$ stacking when the temperature is lowered (Figure S3 of the SI). The increase of the intensity of the $\pi-\pi$ stacking peak is also an indication that the system becomes more ordered (crystalline). ${ }^{4}$ Resulting impact on mobility can be understood with the percolation curves that are superposed on the TI distributions (Figure $1(\mathrm{~d})-(\mathrm{f})) \cdot{ }^{30,47}$ These curves represent the proportion of chains $N(H)$ in the biggest cluster when all chains with a value below a given TI threshold are disconnected, and their sharp increase corresponds to an onset for the transport throughout the whole system. In between $100 \mathrm{~K}$ and $400 \mathrm{~K}$, the $\pi-\pi$ peak decreases only little, and the percolation threshold is only slightly shifted towards lower TI values. As a result, the mobility shows essentially the activated temperature behavior with a positive TC. Then, from $400 \mathrm{~K}$ to $800 \mathrm{~K}$, there is a drastic decrease of the $\pi-\pi$ peak, which even disappears at $800 \mathrm{~K}$. In this range the percolation threshold is largely shifted to lower values of the TI, which results in a drastic mobility decrease with temperature above 400K. Eventually, the complete model $H_{T} \Sigma_{T}$ is a mixture of $H_{300} \Sigma_{T}$ and $H_{T} \Sigma_{300}$ and shows a change in mobility TC from positive to negative around the ambient temperature.

The strong fluctuations of the mobility curves in Figure 2c, d are related to the computational limitations on the size of the MD system investigated. This apparently makes difficult a systematic comparison of calculations with experimental data. (Note there are no such fluctuations in Figure 2a,b because the same MD frame corresponding to $300 \mathrm{~K}$ is used for all temperatures). To overcome this issue, we introduce a generalization of the Gaussian Disorder Model (GDM) usually used to calculate the hopping mobility in organic materials. We start from a GDM already introduced in the literature for PEDOT. ${ }^{44}$ In this model, the transport units are modelled as the sites of a cubic lattice of the size $a$ (see Figure 
3a). Charge carriers are allowed to hop from one site to another site with a hopping rate given by the Miller-Abrahams expression (Equation 1). Energies are drawn from a Gaussian distribution with the broadening $\Sigma$, and the transfer integrals are given by:

$$
H_{i j}=H_{0} \times \exp \left(-\alpha d_{i j}\right),
$$

where $H_{0}$ is a prefactor, $d_{i j}$ is the distance between transport units $i$ and $j$, and $\alpha$ is the inverse localization length, $\alpha=1 / 1.5 a$. Finally, the mobility is calculated by solving the master equation for transport, either via Monte-Carlo, ${ }^{44,48}$ or, as we did here, via a mean-field approach. ${ }^{49}$

Now to incorporate in this model our findings from the multi-scale calculations, we first introduce a temperature dependent $\Sigma(T)$ (Figure 3b) given as previously by $\Sigma(T)=$ $\Sigma_{300}\left(8.88 \times 10^{-4} T+0.73\right)$. In addition, we introduce two inverse localization lengths, $\alpha_{m}$ and $\alpha_{\pi}$, instead of one unique $\alpha$, to model respectively low value TI for misaligned chains and the high value TI for the $\pi-\pi$ stacked chains. This way, the complex TI distribution depicted by the gray area in Figure 3c is approximated by two well defined peaks. We chose $\alpha_{m}=1 / 1.5 a$, same as $\alpha$ in the standard model, and $\alpha_{\pi}=0.5 \alpha_{m}$ corresponding to the ratio between the location of the two peaks in Figure $1(\mathrm{~d})-(\mathrm{f})(\log (|H / \mathrm{eV}|) \simeq-10$ and -5 for misaligned and $\pi-\pi$ configurations respectively). $p_{m}(T)$ and $p_{\pi}(T)$ are the proportion of links with inverse localization length $\alpha_{m}$ and $\alpha_{\pi}$ in the system, as illustrated by the blue and red links in Figure 3a. Nature of a link, i.e. $\alpha_{m}$ or $\alpha_{\pi}$, is simply chosen randomly with the probabilities $p_{m}(T)$ and $p_{\pi}(T)\left(p_{m}(T)+p_{\pi}(T)=1\right)$. Results of this generalized GDM model compare well with results based on MD simulations for four different versions of the model of the mobility calculation, as shown in Figure 2. To model the local alignment of PEDOT chains when the temperature is lowered as observed with MD, the probability $p_{\pi}(T)$ was allowed to change with temperature, and we found that the empirical relationship $p_{\pi}(T)=p_{\pi, 300}\left(1.056-1.056 \times 10^{-6} T^{3}\right)$ can reproduce the MD mobility curves $\mu(T)$ of models $H_{T} \Sigma_{300}$ and $H_{T} \Sigma_{T}$. It is noteworthy that for the comparison to the experiment our 
generalized GDM model has the same number of fitting parameters as the standard one, because the relation between some of the parameters (e.g. the relation between the inverse localization lengths $\alpha_{m}$ and $\alpha_{\pi}$, the temperature dependencies of the probabilities $p(T)$ and the broadening $\Sigma(T)$ ) are obtained from the fitting to the results of the exact multi-scale calculations. Eventually, we have applied this model to reproduce transport properties reported by Weathers et al. ${ }^{50}$ in PEDOT:TOS as shown in Figure 3d (more details in the SI) in order to demonstrate its ability to reproduce both the transition temperature for mobility and the monotonous increase of the Seebeck coefficient with temperature.

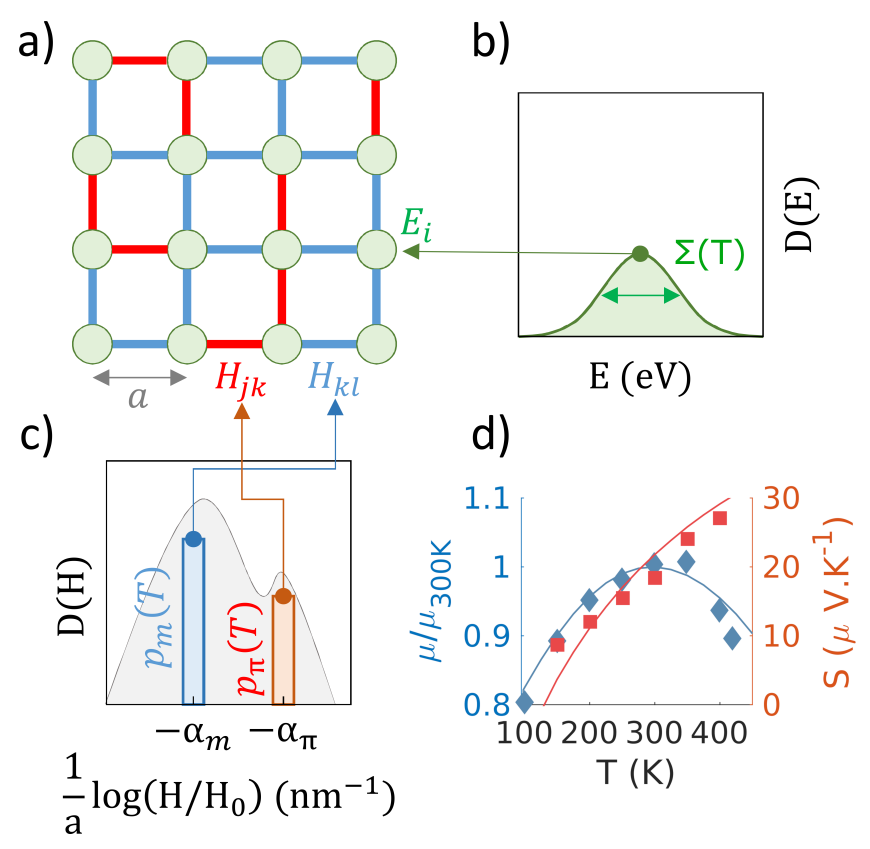

Figure 3: (a) 2D grid of transport units with energies drawn from a temperature-dependent Gaussian distribution and (b) and with transfer integrals drawn from a temperaturedependent bimodal distribution shown in (c). (d) Symbols show experimental measurements of mobility and Seebeck coefficient in PEDOT:TOS reported by Weathers et al. ${ }^{50}$ Solid lines are result from the generalized GDM calculations. $a=1 \mathrm{~nm}, \Sigma(T)=0.1 \mathrm{eV}$.

Finally, it is important to mention that recently, the work of Nan et al. ${ }^{51}$ on Quantum Nuclear Tunnelling (QNT) has reconciled a hopping transport model with the negative mobility temperature coefficient occurring in crystalline organic materials, rubrene and tetracene in this case. This QNT model assumes fully localized charges, similarly to the classical Marcus model of hopping transport (which actually appears as a limit case of the QNT), but includes 
a proper quantum treatment of the phonon heat bath where the quantum nuclear vibrations can affect charge transfer. Jiang et al. ${ }^{52}$ later on demonstrated the predictive power of the QNT compared to Marcus model and band transport model when calculating the mobility in crystalline rubrene, DATT, DNTT, and PDIF-CN2. However, the QNT applied to a semiconducting polymer with a mostly amorphous structure (i.e. the most relevant to the one considered in our study) does not show the negative mobility temperature coefficient. (Note that the above model gives really good quantitative agreement with experiments ${ }^{53,54}$ ). Therefore, it is not clear whether or not the QNT effect could give rise to a negative mobility temperature coefficient in PEDOT:TOS. While recognizing the possible importance of the effect, in this study we focus solely on the effects of the temperature on the distribution of site energies and transfer integrals. A complete model of charge transport should probably combine all these aspects together, which can be an interesting subject for further studies.

\section{Conclusion}

Our results demonstrate that a negative mobility temperature coefficient in PEDOT:TOS at high temperatures can be explained within a hopping transport model, without invoking any assumption about the transition to metallic band-like transport, provided that the evolution of the density of states and the transfer integrals distribution induced by morphological changes when the temperature is increased are taken into account. An extension of the GDM has been proposed to model these phenomena, and it has been shown that it can successfully account for experimental results. We envisage that the short chain length of PEDOT $(<20$ monomers) is a major difference with other conducting polymers; instead of forming large crystallites of folded chains well described as truly metallic islands, ${ }^{21}$ PEDOT typically forms local $\pi-\pi$ stacking, yet extending to an efficient percolation network. These fundamental insights into the origin of the mobility temperature dependence are crucial for electronic devices performance enhancement, particularly for thermoelectric applications. 


\section{Acknowledgement}

This work was supported by KAW foundation (Tail of the Sun), the Swedish Research Council (2016-05990; 2017-04474), and the Advanced Functional Material center at Linköping University. The computations were performed on resources provided by the Swedish National Infrastructure for Computing (SNIC) at NSC and HPC2N.

\section{Supporting Information Available}

The following files are available free of charge.

- Supplementary.pdf: Details on the Molecular Dynamics simulations, charge mobility calculations, Seebeck coefficient calculations, estimation of the DOS broadening with temperature, morphology evolution with temperature and comparison with experimental data.

\section{References}

(1) Forrest, S. R. The Path to Ubiquitous and Low-Cost Organic Electronic Appliances on Plastic. Nature 2004, 428, 911-918.

(2) Kim, N.; Petsagkourakis, I.; Chen, S.; Berggren, M.; Crispin, X.; Jonsson, M. P.; Zozoulenko, I. V. In Conjugated Polymers: Properties, Processing, and Applications, crc press ed.; Reynolds, J. R., Thompson, B. C., Skotheim, T. A., Eds.; 2019; Vol. 1.

(3) Wijeratne, K.; Vagin, M.; Brooke, R.; Crispin, X. Poly(3,4-Ethylenedioxythiophene)Tosylate (PEDOT-Tos) Electrodes in Thermogalvanic Cells. J. Mater. Chem. A 2017, 5, 19619-19625.

(4) Petsagkourakis, I.; Pavlopoulou, E.; Portale, G.; Kuropatwa, B. A.; Dilhaire, S.; Fleury, G.; Hadziioannou, G. Structurally-Driven Enhancement of Thermoelectric 
Properties within Poly(3,4-Ethylenedioxythiophene) Thin Films. Sci. Rep. 2016, 6, 30501.

(5) Bubnova, O.; Berggren, M.; Crispin, X. Tuning the Thermoelectric Properties of Conducting Polymers in an Electrochemical Transistor. J. Am. Chem. Soc. 2012, 134, $16456-16459$.

(6) Nardes, A. M.; Kemerink, M.; Janssen, R. A. J. Anisotropic Hopping Conduction in Spin-Coated PEDOT:PSS Thin Films. Phys. Rev. B 2007, 76, 085208.

(7) Nardes, A. M.; Janssen, R. A. J.; Kemerink, M. A Morphological Model for the SolventEnhanced Conductivity of PEDOT:PSS Thin Films. Adv. Funct. Mater. 2008, 18, $865-871$.

(8) Kim, N.; Lee, B. H.; Choi, D.; Kim, G.; Kim, H.; Kim, J.-R.; Lee, J.; Kahng, Y. H.; Lee, K. Role of Interchain Coupling in the Metallic State of Conducting Polymers. Phys. Rev. Lett. 2012, 109, 106405.

(9) Yu, Z.; Xia, Y.; Du, D.; Ouyang, J. PEDOT:PSS Films with Metallic Conductivity through a Treatment with Common Organic Solutions of Organic Salts and Their Application as a Transparent Electrode of Polymer Solar Cells. ACS Appl. Mater. Interfaces 2016, 8, 11629-11638.

(10) Mott, N. F.; Davis, E. A. Electronic Processes in Non-Crystalline Materials; OUP Oxford, 2012.

(11) MacDiarmid, A. G.; Epstein, A. J. The Concept of Secondary Doping as Applied to Polyaniline. Synth. Met. 1994, 65, 103-116.

(12) Ahlskog, M.; Reghu, M.; Heeger, A. J. The Temperature Dependence of the Conductivity in the Critical Regime of the Metal - Insulator Transition in Conducting Polymers. J. Phys.: Condens. Matter 1997, 9, 4145-4156. 
(13) Sheng, P. Fluctuation-Induced Tunneling Conduction in Disordered Materials. Phys. Rev. B 1980, 21, 2180-2195.

(14) Farka, D.; Jones, A. O. F.; Menon, R.; Sariciftci, N. S.; Stadler, P. Metallic Conductivity beyond the Mott Minimum in PEDOT: Sulphate at Low Temperatures. Synth. Met. 2018, 240, 59-66.

(15) Massonnet, N.; Carella, A.; de Geyer, A.; Faure-Vincent, J.; Simonato, J.-P. Metallic Behaviour of Acid Doped Highly Conductive Polymers. Chem. Sci. 2015, 6, 412-417.

(16) Kumar, S. R. S.; Kurra, N.; Alshareef, H. N. Enhanced High Temperature Thermoelectric Response of Sulphuric Acid Treated Conducting Polymer Thin Films. J. Mater. Chem. C 2015, 4, 215-221.

(17) Yu, Z.; Xia, Y.; Du, D.; Ouyang, J. PEDOT:PSS Films with Metallic Conductivity through a Treatment with Common Organic Solutions of Organic Salts and Their Application as a Transparent Electrode of Polymer Solar Cells. ACS Appl. Mater. Interfaces 2016, 8, 11629-11638.

(18) Paziresh, N.; Sotzing, G. A. Semiconductor-Metal Transition in Poly(3,4Ethylenedioxythiophene): Poly(Styrenesulfonate) and Its Electrical Conductivity While Being Stretched. Polym. Eng. Sci. 2019, 59, 1051.

(19) Rudd, S.; Franco-Gonzalez, J. F.; Singh, S. K.; Khan, Z. U.; Crispin, X.; Andreasen, J. W.; Zozoulenko, I.; Evans, D. Charge Transport and Structure in Semimetallic Polymers. J. Polym. Sci., Part B: Polym. Phys. 2018, 56, 97-104.

(20) Gueye, M. N.; Carella, A.; Massonnet, N.; Yvenou, E.; Brenet, S.; Faure-Vincent, J.; Pouget, S.; Rieutord, F.; Okuno, H.; Benayad, A.; Demadrille, R.; Simonato, J.-P. Structure and Dopant Engineering in PEDOT Thin Films: Practical Tools for a Dramatic Conductivity Enhancement. Chem. Mater. 2016, 28, 3462-3468. 
(21) Kaiser, A. B. Systematic Conductivity Behavior in Conducting Polymers: Effects of Heterogeneous Disorder. Adv. Mater. 2001, 13, 927-941.

(22) Bubnova, O.; Khan, Z. U.; Wang, H.; Braun, S.; Evans, D. R.; Fabretto, M.; HojatiTalemi, P.; Dagnelund, D.; Arlin, J.-B.; Geerts, Y. H.; Desbief, S.; Breiby, D. W.; Andreasen, J. W.; Lazzaroni, R.; Chen, W. M.; Zozoulenko, I.; Fahlman, M.; Murphy, P. J.; Berggren, M.; Crispin, X. Semi-Metallic Polymers. Nat. Mater. 2014, 13, 190-194.

(23) Wang, X.; Zhang, X.; Sun, L.; Lee, D.; Lee, S.; Wang, M.; Zhao, J.; Shao-Horn, Y.; Dincă, M.; Palacios, T.; Gleason, K. K. High Electrical Conductivity and Carrier Mobility in oCVD PEDOT Thin Films by Engineered Crystallization and Acid Treatment. Sci. Adv. 2018, 4, eaat5780.

(24) Ashcroft, N. W.; Mermin, N. D. Solid State Physics.; Saunders college: Philadelphia, Pa., 1976; OCLC: 472084776.

(25) Beenakker, C. W. J.; van Houten, H. In Solid State Physics; Ehrenreich, H., Turnbull, D., Eds.; Semiconductor Heterostructures and Nanostructures; Academic Press, 1991; Vol. 44; pp 1-228.

(26) Kang, K.; Watanabe, S.; Broch, K.; Sepe, A.; Brown, A.; Nasrallah, I.; Nikolka, M.; Fei, Z.; Heeney, M.; Matsumoto, D.; Marumoto, K.; Tanaka, H.; Kuroda, S.-I.; Sirringhaus, H. 2D Coherent Charge Transport in Highly Ordered Conducting Polymers Doped by Solid State Diffusion. Nat. Mater. 2016, 15, 896-902.

(27) Kaiser, A. B. Metallic Behaviour in Highly Conducting Polymers. Synth. Met. 1991, $45,183-196$.

(28) Elschner, A.; Kirchmeyer, S.; Lovenich, W.; Merker, U.; Reuter, K.; Kirchmeyer, S.; Lovenich, W.; Merker, U.; Reuter, K. PEDOT : Principles and Applications of an Intrinsically Conductive Polymer; CRC Press, 2010. 
(29) Ugur, A.; Katmis, F.; Li, M.; Wu, L.; Zhu, Y.; Varanasi, K. K.; Gleason, K. K. LowDimensional Conduction Mechanisms in Highly Conductive and Transparent Conjugated Polymers. Adv. Mater. 2015, 27, 4604-4610.

(30) Rolland, N.; Franco-Gonzalez, J. F.; Volpi, R.; Linares, M.; Zozoulenko, I. V. Understanding Morphology-Mobility Dependence in PEDOT:Tos. Phys. Rev. Mater. 2018, 2, 045605 .

(31) Rühle, V.; Kirkpatrick, J.; Andrienko, D. A Multiscale Description of Charge Transport in Conjugated Oligomers. J. Chem. Phys. 2010, 132, 134103.

(32) Vehoff, T.; Baumeier, B.; Troisi, A.; Andrienko, D. Charge Transport in Organic Crystals: Role of Disorder and Topological Connectivity. J. Am. Chem. Soc. 2010, 132, $11702-11708$.

(33) Rühle, V.; Lukyanov, A.; May, F.; Schrader, M.; Vehoff, T.; Kirkpatrick, J.; Baumeier, B.; Andrienko, D. Microscopic Simulations of Charge Transport in Disordered Organic Semiconductors. J. Chem. Theory Comput. 2011, 7, 3335-3345.

(34) Jakobsson, M.; Linares, M.; Stafström, S. Monte Carlo Simulations of Charge Transport in Organic Systems with True Off-Diagonal Disorder. J. Chem. Phys. 2012, 137, 114901.

(35) Volpi, R.; Stafström, S.; Linares, M. Transition Fields in Organic Materials: From Percolation to Inverted Marcus Regime. A Consistent Monte Carlo Simulation in Disordered PPV. J. Chem. Phys. 2015, 142, 094503.

(36) Franco-Gonzalez, J. F.; Rolland, N.; Zozoulenko, I. V. Substrate-Dependent Morphology and Its Effect on Electrical Mobility of Doped Poly(3,4-Ethylenedioxythiophene) (PEDOT) Thin Films. ACS Appl. Mater. Interfaces 2018, 10, 29115-29126. 
(37) Miller, E. D.; Jones, M. L.; Jankowski, E. Tying Together Multiscale Calculations for Charge Transport in P3HT: Structural Descriptors, Morphology, and Tie-Chains. Polymers 2018, 10, 1358.

(38) Franco-Gonzalez, J. F.; Zozoulenko, I. V. Molecular Dynamics Study of Morphology of Doped PEDOT: From Solution to Dry Phase. J. Phys. Chem. B 2017, 121, 4299-4307.

(39) Modarresi, M.; Felipe Franco-Gonzalez, J.; Zozoulenko, I. Computational Microscopy Study of the Granular Structure and pH Dependence of PEDOT:PSS. Phys. Chem. Chem. Phys. 2019, 21, 6699-6711.

(40) Felipe Franco-Gonzalez, J.; Pavlopoulou, E.; Stavrinidou, E.; Gabrielsson, R.; T. Simon, D.; Berggren, M.; V. Zozoulenko, I. Morphology of a Self-Doped Conducting Oligomer for Green Energy Applications. Nanoscale 2017, 9, 13717-13724.

(41) Mehandzhiyski, A. Y.; Zozoulenko, I. Computational Microscopy of PEDOT:PSS/Cellulose Composite Paper. ACS Appl. Energy Mater. 2019, 2, 3568-3577.

(42) Miller, A.; Abrahams, E. Impurity Conduction at Low Concentrations. Phys. Rev. A 1960, 120, 745-755.

(43) Yu, Z. G.; Smith, D. L.; Saxena, A.; Martin, R. L.; Bishop, A. R. Molecular Geometry Fluctuations and Field-Dependent Mobility in Conjugated Polymers. Phys. Rev. B 2001, 63, 085202.

(44) Ihnatsenka, S.; Crispin, X.; Zozoulenko, I. V. Understanding Hopping Transport and Thermoelectric Properties of Conducting Polymers. Phys. Rev. B 2015, 92, 035201.

(45) Muñoz, W. A.; Singh, S. K.; Franco-Gonzalez, J. F.; Linares, M.; Crispin, X.; Zozoulenko, I. V. Insulator to Semimetallic Transition in Conducting Polymers. Phys. Rev. B 2016, 94, 205202. 
(46) Muñoz, W. A.; Crispin, X.; Fahlman, M.; Zozoulenko, I. V. Understanding the Impact of Film Disorder and Local Surface Potential in Ultraviolet Photoelectron Spectroscopy of PEDOT. Macromol. Rapid Commun. 2018, 39, 1700533.

(47) Savoie, B. M.; Kohlstedt, K. L.; Jackson, N. E.; Chen, L. X.; de la Cruz, M. O.; Schatz, G. C.; Marks, T. J.; Ratner, M. A. Mesoscale Molecular Network Formation in Amorphous Organic Materials. Proc. Natl. Acad. Sci. U.S.A. 2014, 111, 10055-10060.

(48) Bässler, H. Charge Transport in Disordered Organic Photoconductors a Monte Carlo Simulation Study. Phys. Status Solidi B 1993, 175, 15-56.

(49) Cottaar, J.; Bobbert, P. A. Calculating Charge-Carrier Mobilities in Disordered Semiconducting Polymers: Mean Field and Beyond. Phys. Rev. B 2006, 74, 115204.

(50) Weathers, A.; Khan, Z. U.; Brooke, R.; Evans, D.; Pettes, M. T.; Andreasen, J. W.; Crispin, X.; Shi, L. Significant Electronic Thermal Transport in the Conducting Polymer Poly(3,4-Ethylenedioxythiophene). Adv. Mater. 2015, 27, 2101-2106.

(51) Nan, G.; Yang, X.; Wang, L.; Shuai, Z.; Zhao, Y. Nuclear Tunneling Effects of Charge Transport in Rubrene, Tetracene, and Pentacene. Phys. Rev. B 2009, 79, 115203.

(52) Jiang, Y.; Geng, H.; Li, W.; Shuai, Z. Understanding Carrier Transport in Organic Semiconductors: Computation of Charge Mobility Considering Quantum Nuclear Tunneling and Delocalization Effects. J. Chem. Theory Comput. 2019, 15, 1477-1491.

(53) Asadi, K.; Kronemeijer, A. J.; Cramer, T.; Jan Anton Koster, L.; Blom, P. W. M.; de Leeuw, D. M. Polaron Hopping Mediated by Nuclear Tunnelling in Semiconducting Polymers at High Carrier Density. Nat. Commun. 2013, 4, 1710.

(54) van der Kaap, N. J.; Katsouras, I.; Asadi, K.; Blom, P. W. M.; Koster, L. J. A.; de Leeuw, D. M. Charge Transport in Disordered Semiconducting Polymers Driven by Nuclear Tunneling. Phys. Rev. B 2016, 93, 140206. 


\section{Graphical TOC Entry}

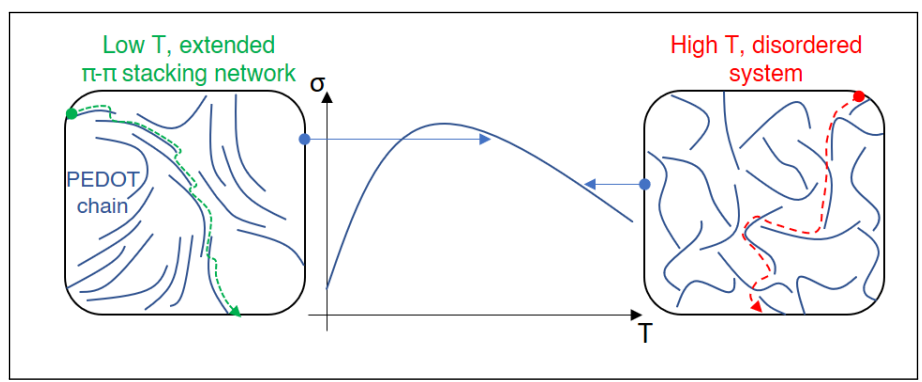




\title{
Supporting Information:
}

\section{Can Mobility Negative Temperature}

Coefficient Be Reconciled with the Hopping

Character of Transport in Conducting

\section{Polymers?}

\author{
Nicolas Rolland, ${ }^{\dagger, \ddagger}$ Juan Felipe Franco-Gonzalez, ${ }^{\ddagger}$ and Igor Zozoulenko*, \\ †Division of Theoretical Chemistry 8 Biotechnology, Royal Institute of Technology (KTH), \\ SE-10691 Stockholm, Sweden \\ $\ddagger$ Laboratory of Organic Electronics, ITN, Linköping University, SE-601 74 Norrköping, \\ Sweden \\ *E-mail: igor.zozoulenko@liu.se
}




\section{S1. Molecular Dynamics Simulations}

We investigated the transport properties in PEDOT thin films doped with TOS counterions. The bulk morphology of this system was generated by atomistic Molecular Dynamics (MD) using LAMMPS software package ${ }^{\mathrm{S} 1}$ and the parameters for each atom were taken from AMBER general force field (GAFF) similar to the one of our previous work. ${ }^{\text {S2 }}$ Note that practically the same morphology is obtained using the OPLS-AA force field. 160 PEDOT chains with $N=12$ monomers were randomly placed in a cubic simulation box of size $8 \mathrm{~nm}$, with periodic boundary conditions in all directions. Each PEDOT chain carries an excess positive charge $+4 e$ to mimic the $33 \%$ oxidation level, which is typical for pristine (i.e. as polymerized PEDOT), and the system was kept neutral overall by randomly introducing 640 TOS ions each carrying a charge $-e$. The resulting atomic charges are calculated with the first-principles density-functional theory functional WB97XD ${ }^{\mathrm{S} 3}$ with the $6-31+\mathrm{g}(\mathrm{d})^{\mathrm{S} 4}$ basis set as implemented in Gaussian 09. ${ }^{\text {S5 }}$ The partial charge per atom was taken from the fitting to electrostatic potential population analysis (ESP). ${ }^{\mathrm{S} 6}$ Note that for all oxidation levels the charges are distributed rather homogeneously over a polymer chain. As a result,

the resulting morphology is only weakly sensitive to the charging of the polymer chains. ${ }^{\mathrm{S} 2}$ The system was equilibrated during 500ps at $800 \mathrm{~K}$, keeping the pressure and temperature constant (nPT) with both isotropic-barostat and thermostat as Nose-Hoover, and the temperature was then cooled down from $800 \mathrm{~K}$ to $100 \mathrm{~K}$ at a rate of $10 \mathrm{~K} / 500 \mathrm{ps}$, with $500 \mathrm{ps}$ of equilibration for each temperature. 15 frames were extracted from this simulation, corresponding to equilibrated systems at temperature ranging from $800 \mathrm{~K}$ to $100 \mathrm{~K}$ with a step of $50 \mathrm{~K}(800 \mathrm{~K}, 750 \mathrm{~K}, 700 \mathrm{~K}, 650 \mathrm{~K}, 600 \mathrm{~K}, 550 \mathrm{~K}, 500 \mathrm{~K}, 450 \mathrm{~K}, 400 \mathrm{~K}, 350 \mathrm{~K}, 300 \mathrm{~K}, 250 \mathrm{~K}, 200 \mathrm{~K}$, 150K, 100K). Typical snaphots of these equilibrated systems are reported in Figure S1. This procedure was repeated for 4 different realisations of the initial simulation box (i.e. random positions of the molecules in the box) to reduce statistical fluctuations. The XRD diffraction patterns were calculated on the resulting morphologies as implemented in LAMMPS. ${ }^{\mathrm{S}, \mathrm{S} 7}$ 

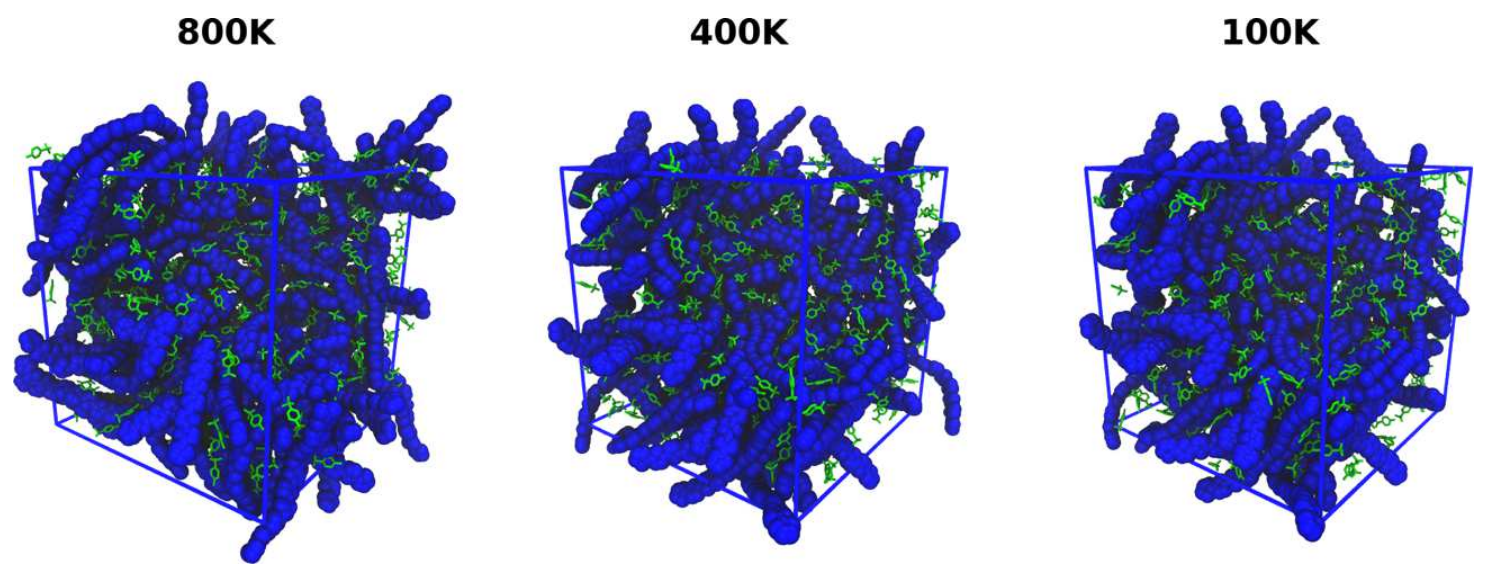

Figure S1: Snapshots of MD simulation of PEDOT:TOS from 800K to 100K. Blue spheres represent the atoms forming the thiophene rings of the PEDOT chains (4 Carbon atoms and 1 Sulfur atom form a thiophene ring for each EDOT monomer). The green sticks represent TOS molecules, where hydrogens are not shown for clarity.

\section{S2. Charge carrier mobility}

Each frame produced by MD (4 realizations x 15 frames $=60$ frames in total) was analyzed and transport properties extracted following the method developed in our previous works. ${ }^{\text {S8,S9 }}$ First, a resistive network is built by considering that 4 transport units, that can each accommodate one charge carrier, are located at the center of each PEDOT chain. Then, transport units in the box are linked if their distance is below a threshold $d_{t}=8 \AA$ (note that transport units on the same chain are automatically linked). Each link corresponds to a possible carrier hop from one transport unit to another with a hopping rate given by the Miller-Abrahams formalism:

$$
\omega_{i j}=\omega_{0} \times\left|H_{i j}\right|^{2} \times \exp \left(-\frac{E_{j}-E_{i}+\left|E_{j}-E_{i}\right|-2 e \vec{F} \cdot \vec{r}}{2 k_{B} T}\right)
$$

where $\omega_{0}$ is a pre-factor, $H_{i j}$ is the transfer integral between molecular orbitals representing the localized states $i$ and $j, E_{i}$ and $E_{j}$ are the states energies, randomly drawn from a Gaussian distribution with the broadening $\Sigma, \vec{F}$ is the applied electric field and $\overrightarrow{r_{i j}}$ is the vector between sites $i$ and $j$. For calculation of the transfer integral $H_{i j}$ between transport units belonging to different chains, we made the approximation that it can be treated as the 
transfer integral between the molecular orbitals (MOs) of the neutral states of the chains. We use the dimer projection method at the ZINDO level ${ }^{\mathrm{S} 10}$ where all corresponding MOs are calculated using GAUSSIAN09 package $^{\mathrm{S} 5}$ for PEDOT chains as obtained from the MD simulations; the transfer integrals are evaluated for the neutral chains for the 4 highest MOs in the valence band. For transport units on the same chain, we simply set the transfer integral to $1 \mathrm{eV}$. Regarding the transport units energies $E_{i}$, we simply drawn them from a Gaussian distribution with the broadening $\Sigma$. Note that we also had access to the chain MOs energies as given by GAUSSIAN. Finally, mobility in the system is determined by solving the Master Equation for transport in a mean field fashion as introduced by Yu et al., ${ }^{\mathrm{S} 11}$ applying a field $F=10^{4} \mathrm{~V} / \mathrm{cm}^{-1}$ and considering a carrier concentration $c=2.5 \times 10^{20} \mathrm{~cm}^{-3}$ (mobility values are averaged over multiple Gaussian DOS realizations and field directions). This Master Equation describes the probability $p_{i}$ for each transport unit to be occupied by a charge carrier in the stationary state:

$$
\sum_{i \neq j}\left[-\omega_{i j} p_{i}\left(1-p_{j}\right)+\omega_{j i} p_{j}\left(1-p_{i}\right)\right]=0
$$

When the equation is solved and all $p_{i}$ are know, the mobility can be directly calculated:

$$
\mu=\frac{\sum_{i, j, i \neq j} \omega_{i j} p_{i}\left(1-p_{j}\right) \vec{F} \cdot \overrightarrow{r_{i j}}}{p N F^{2}}
$$

where $p$ is the average occupation probability and $N$ is the number of transport units in the system. Note that in our study we only report relative values of mobility because we do not know the value of the prefactor $\omega_{0}$ appearing in Equation 1.

\section{S3. Seebeck coefficient}

The Seebeck coefficient was calculated following the method used by Upadhyahya et al. ${ }^{\mathrm{S} 12}$ It is expressed as: 


$$
S(T)=\frac{E_{F}-E_{\text {Trans }}}{|e| T}
$$

where $E_{F}$ is the Fermi level, $E_{\text {Trans }}$ is the transport energy, $e$ is the electron charge and $T$ is the temperature. The Fermi level is obtained from the charge carrier concentration and indirectly depends on the shape of the density of states via the Fermi-Dirac distribution:

$$
p=\frac{\sum_{i} p_{i}^{0}}{N}=\frac{1}{N} \sum_{i} \frac{1}{1+\exp \left(\frac{E_{i}-E_{F}}{k_{B} T}\right)},
$$

where $p_{i}^{0}$ is the initial occupation probability of transport unit $i$ (before solving the Master Equation for transport). Once the Master Equation is solved and the $p_{i}$ are known, the transport energy can be calculated by evaluating the contribution of each energy to the total current:

$$
E_{T}=\frac{\sum_{i, j} E_{i} \omega_{i j} p_{i}\left(1-p_{j}\right) \vec{F} \cdot \overrightarrow{r_{i j}}}{\sum_{i, j} \omega_{i j} p_{i}\left(1-p_{j}\right) \vec{F} \cdot \overrightarrow{r_{i j}}}
$$

Note that there are no prefactor left when calculating the Seebeck coefficient, and reported values are thus absolute values.

\section{S4. Estimation of the Density of States broadening with temperature}

We do not direct access to the Density Of States due to the prohibitive size of our system for a full QM calculation. It is generally accepted that the DOS can be approximated by a gaussian distribution with typical standard deviation $0.1 \mathrm{eV},{ }^{\mathrm{S} 13, \mathrm{~S} 14}$ thus we set $\Sigma_{300}=\Sigma(T=300 K)=$ $0.1 \mathrm{eV}$. To account for the DOS evolution due to the PEDOT chain conformations evolving with temperature, we calculated how the distribution of HOMO energy levels evolves in our system. To do so, we simply performed QM calculations on every single neutral PEDOT 
chains extracted from the MD simulations, we built the histogram of these energies and fit them with a gaussian distribution. The broadening of these Gaussian fits as a function of temperature is reported in Figure $\mathrm{S} 2$.

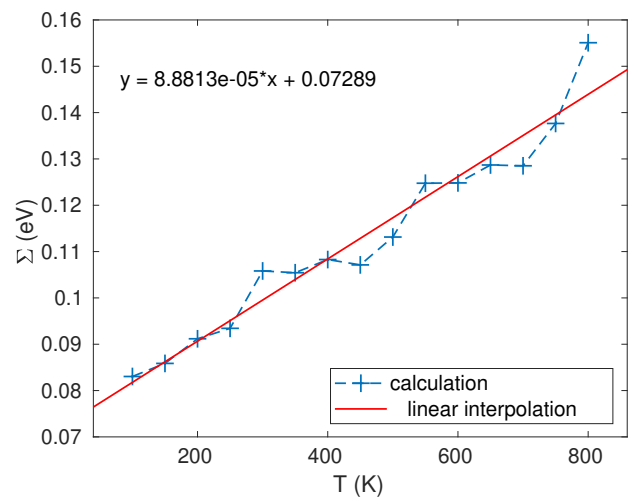

Figure S2: Temperature evolution of the broadening $\Sigma$ of the Gaussian fit to the HOMO energies distribution.

From this plot, we have extracted the value $d \Sigma_{H O M O} / d T$ and we have then set $d \Sigma / d T=$ $d \Sigma_{\text {HOMO }} / d T$. Finally, combining the know value $\Sigma_{300}$ and the approximation $d \Sigma / d T=$ $d \Sigma_{\text {HOMO }} / d T$, we end up with the relation $\Sigma(T)=\Sigma_{300}\left(8.88 \times 10^{-4} T+0.73\right)$.

\section{S5. Morphology and transfer integral distributions evolu- tion with temperature}

The correlation between XRD diffraction patterns (Figure S3a) and the transfer integrals distributions shown in the main manuscript clearly indicates a number of $\pi-\pi$ stacking configurations increases with decreasing temperature. We attribute these morphological changes to a local reorganization of the chains during the cooling: overall, the global morphology is unchanged with temperature, but locally chains that are close to each other can reorganize to form well-defined $\pi-\pi$ stacking when the temperature is lowered. This is confirmed by the examination of the geometry versus transfer integral plots in Figure S3c,d, where $R_{M-M}$ is the distance between the centers of the closest monomers $i$ and $j$ of a pair of 
PEDOT chains and $S_{M-M}$ is a stacking factor defined as $S_{M-M}=\left|\overrightarrow{n_{i}} \cdot \overrightarrow{r_{i j}}\right| \cdot\left|\overrightarrow{n_{j}} \cdot \overrightarrow{r_{i j}}\right|$, where $\overrightarrow{n_{i}}$ and $\overrightarrow{n_{j}}$ are normal unit vectors of the monomers $i$ and $j$ and $\overrightarrow{r_{i j}}$ is the vector linking centers of monomers $i$ and $j$. Apparently, the stacking factor $S_{M-M}=1$ for completely aligned monomers, and $S_{M-M}=0$ for misaligned ones. The color map indicates the transfer integral associated to each configuration. Between $800 \mathrm{~K}$ and $100 \mathrm{~K}$ the scatter plots show only a local reorganization corresponding to a concentration of high transfer integrals points around $S_{M-M}=1$ and $R_{M-M}=r_{\pi} \simeq 3.5 \AA$, signature of a local alignment of chains into $\pi-\pi$ stacking configuration.

a)

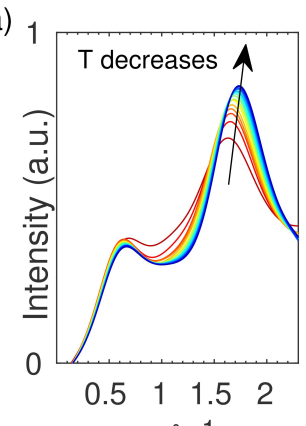

b)

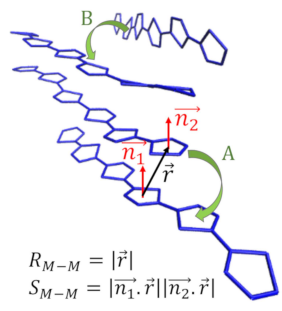

c)

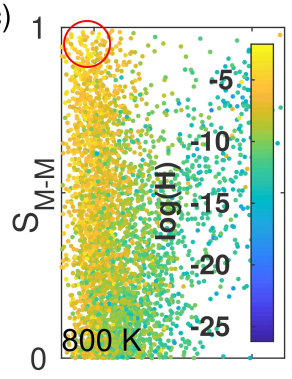

d)

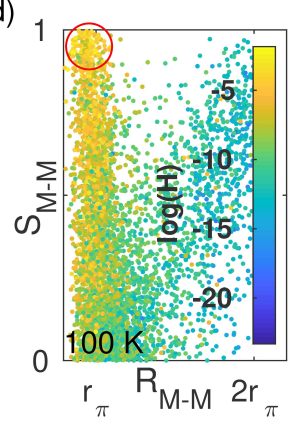

Figure S3: a) XRD diffraction patterns as a function of temperature, from $800 \mathrm{~K}$ (red curve) to $100 \mathrm{~K}$ (blue curve). b) Definition of geometrical parameters on a chain with 6 monomers, only the chain backbone is represented. $\mathrm{c}, \mathrm{d}$ ) Transfer integral versus geometry plots for $\mathrm{T}$ $=100 \mathrm{~K}$ and $\mathrm{T}=800 \mathrm{~K}$. 


\section{S6. Application of the model to Weathers et al. experi- mental results}

As a validation of our model we have reproduced the experimental results by Weathers et al. ${ }^{\text {S15 }}$ reporting both conductivity and Seebeck coefficient as a function of temperature of PEDOT:TOS. In particular, we focus on the sample S1 of this study because it exhibits a transition temperature from a negative to a positive conductivity temperature coefficient. Note that our results are expressed in term of scaled mobility, $\mu(T) / \mu(300 K)$, while the experimental curves report values of the scaled conductivity $\sigma(T) / \sigma(300 K)$. Both can be directly compared because in PEDOT the charge carrier concentration $n$ is constant with temperature, and $\sigma=q n \mu$. The parameters we found to reproduce the experimental results are $\Sigma_{300}=1.05 \mathrm{eV}$ and $p=0.24$ (charge concentration per transport units) for a field $F=10^{5} \mathrm{Vcm}^{-1}$.

\section{References}

(S1) Plimpton, S. Fast Parallel Algorithms for Short-Range Molecular Dynamics. J. Comput. Phys. 1995, 117, 1-19.

(S2) Franco-Gonzalez, J. F.; Zozoulenko, I. V. Molecular Dynamics Study of Morphology of Doped PEDOT: From Solution to Dry Phase. J. Phys. Chem. B 2017, 121, 42994307.

(S3) Lin, Y.-S.; Li, G.-D.; Mao, S.-P.; Chai, J.-D. Long-Range Corrected Hybrid Density Functionals with Improved Dispersion Corrections. J. Chem. Theory Comput. 2013, 9, 263-272.

(S4) Krishnan, R.; Binkley, J. S.; Seeger, R.; Pople, J. A. Self-consistent Molecular Orbital 
Methods. XX. A Basis Set for Correlated Wave Functions. J. Chem. Phys. 1980, 72, $650-654$.

(S5) Frisch, M. J.; Trucks, G. W.; Schlegel, H. B.; Scuseria, G. E.; Robb, M. A.; Cheeseman, J. R.; Scalmani, G.; Barone, V.; Mennucci, B.; Petersson, G. A.; Nakatsuji, H.; Caricato, M.; Li, X.; Hratchian, H. P.; Izmaylov, A. F.; Bloino, J.; Zheng, G.; Sonnenberg, J. L.; Hada, M.; Ehara, M.; Toyota, K.; Fukuda, R.; Hasegawa, J.; Ishida, M.; Nakajima, T.; Honda, Y.; Kitao, O.; Nakai, H.; Vreven, T.; Montgomery, J. A., Jr.; Peralta, J. E.; Ogliaro, F.; Bearpark, M.; Heyd, J. J.; Brothers, E.; Kudin, K. N.; Staroverov, V. N.; Kobayashi, R.; Normand, J.; Raghavachari, K.; Rendell, A.; Burant, J. C.; Iyengar, S. S.; Tomasi, J.; Cossi, M.; Rega, N.; Millam, J. M.; Klene, M.; Knox, J. E.; Cross, J. B.; Bakken, V.; Adamo, C.; Jaramillo, J.; Gomperts, R.; Stratmann, R. E.; Yazyev, O.; Austin, A. J.; Cammi, R.; Pomelli, C.; Ochterski, J. W.; Martin, R. L.; Morokuma, K.; Zakrzewski, V. G.; Voth, G. A.; Salvador, P.; Dannenberg, J. J.; Dapprich, S.; Daniels, A. D.; Farkas, O.; Foresman, J. B.; Ortiz, J. V.; Cioslowski, J.; Fox, D. J. Gaussian 09 Revision E.01. Gaussian Inc. Wallingford CT 2009.

(S6) Singh, U. C.; Kollman, P. A. An Approach to Computing Electrostatic Charges for Molecules. J. Comput. Chem. 1984, 5, 129-145.

(S7) Coleman, S. P.; Spearot, D. E.; Capolungo, L. Virtual Diffraction Analysis of Ni [0 1 0] Symmetric Tilt Grain Boundaries. Modell. Simul. Mater. Sci. Eng. 2013, 21, 055020 .

(S8) Rolland, N.; Franco-Gonzalez, J. F.; Volpi, R.; Linares, M.; Zozoulenko, I. V. Understanding Morphology-Mobility Dependence in PEDOT:Tos. Phys. Rev. Mater. 2018, 2, 045605 .

(S9) Franco-Gonzalez, J. F.; Rolland, N.; Zozoulenko, I. V. Substrate-Dependent Morphol- 
ogy and Its Effect on Electrical Mobility of Doped Poly(3,4-Ethylenedioxythiophene) (PEDOT) Thin Films. ACS Appl. Mater. Interfaces 2018, 10, 29115-29126.

(S10) Kirkpatrick, J. An Approximate Method for Calculating Transfer Integrals Based on the ZINDO Hamiltonian. Int. J. Quantum Chem. 2008, 108, 51-56.

(S11) Yu, Z. G.; Smith, D. L.; Saxena, A.; Martin, R. L.; Bishop, A. R. Molecular Geometry Fluctuations and Field-Dependent Mobility in Conjugated Polymers. Phys. Rev. B 2001, 63, 085202.

(S12) Upadhyaya, M.; Boyle, C. J.; Venkataraman, D.; Aksamija, Z. Effects of Disorder on Thermoelectric Properties of Semiconducting Polymers. arXiv:1901.03370 [cond-mat] 2019,

(S13) Muñoz, W. A.; Singh, S. K.; Franco-Gonzalez, J. F.; Linares, M.; Crispin, X.; Zozoulenko, I. V. Insulator to Semimetallic Transition in Conducting Polymers. Phys. Rev. B 2016, 94, 205202.

(S14) Muñoz, W. A.; Crispin, X.; Fahlman, M.; Zozoulenko, I. V. Understanding the Impact of Film Disorder and Local Surface Potential in Ultraviolet Photoelectron Spectroscopy of PEDOT. Macromol. Rapid Commun. 2018, 39, 1700533.

(S15) Weathers, A.; Khan, Z. U.; Brooke, R.; Evans, D.; Pettes, M. T.; Andreasen, J. W.; Crispin, X.; Shi, L. Significant Electronic Thermal Transport in the Conducting Polymer Poly(3,4-Ethylenedioxythiophene). Adv. Mater. 2015, 27, 2101-2106. 